\title{
SUBADDITIVE FUNCTIONS AND A RELAXATION OF THE HOMOGENEITY CONDITION OF SEMINORMS
}

\author{
JANUSZ MATKOWSKI
}

(Communicated by Andrew M. Bruckner)

\begin{abstract}
We prove that every locally bounded above at a point subadditive function $f:(0, \infty) \rightarrow \mathbb{R}$ such that $f(r t) \leq r f(t), t>0$, for some $r \in$ $(0,1)$ has to be linear. Using this we show among others that the homogeneity condition of a seminorm $\mathbf{p}$ in a real linear space $X$ can be essentially relaxed to the following condition: there exists an $r \in(0,1)$ such that $\mathbf{p}(r x) \leq r \mathbf{p}(x)$ for all $x \in X$. A new characterization of the $\mathbf{L}^{p}$-norm and one-line proofs of Minkowski's and Hölder's inequalities are also given.
\end{abstract}

\section{INTRODUCTION}

Let $\mathbb{R}, \mathbb{R}_{+}$, and $\mathbb{N}$ denote, respectively, the set of reals, nonnegative reals, and positive integers.

In the first section of this paper we deal with some properties of subadditive and superadditive functions defined in $(a, \infty), a \geq 0$. We say for short that a function $f:(0, \infty) \rightarrow \mathbb{R}$ is locally bounded above at a point if there exist $t_{0}>0, \delta>0$, and $M \in \mathbb{R}$ such that $f(t) \leq M$ for all $t \in\left(t_{0}-\delta, t_{0}+\delta\right)$.

The main result says that every locally bounded above at a point subadditive function $f:(0, \infty) \rightarrow \mathbb{R}$ such that $f(r t) \leq r f(t)$ for some $r \in(0,1)$ and all $t>0$ has to be linear, i.e., $f(t)=f(1) t, t>0$. Using this fact, in $\S 2$ we show that the homogeneity condition in the definition of a seminorm (or a norm) can be replaced by a considerably weaker one. In particular, we prove that if $X$ is a real linear space and $\mathbf{p}: X \rightarrow \mathbb{R}$ satisfies the following three conditions:

(1) $\mathbf{p}(x+y) \leq \mathbf{p}(x)+\mathbf{p}(y)$ for all $x, y \in X$,

(2) $\mathbf{p}(r x) \leq r \mathbf{p}(x)$ for some $r \in(0,1)$ and all $x \in X$; and the function $t \rightarrow \mathbf{p}(t x), t>0$, is locally bounded above at a point

(3) $\mathbf{p}(-x)=\mathbf{p}(x)$ for all $x \in X$, then $\mathbf{p}(t x)=|t| \mathbf{p}(x)$ for all $t \in \mathbb{R}$ and $x \in X$, i.e., $\mathbf{p}$ is a seminorm.

Actually conditions (1) and (2) can be replaced by the following more general one: for every $x \in X$ the function $f_{x}:(0, \infty) \rightarrow \mathbb{R}$, defined by the formula

Received by the editors February 13, 1991 and, in revised form, July 8, 1991; the main results of this paper have been presented to the 28th International Symposium on Functional Equations, Graz-Mariatrost, Austria, August 23-September 1, 1990.

1991 Mathematics Subject Classification. Primary 26D10, 26A51, 39C05, 46E30.

Key words and phrases. Subadditive functions, seminorm, measure space, characterization of $\mathbf{L}^{p}$-norm, Minkowski's inequality, Hölder's inequality. 
$f_{x}(t):=\mathbf{p}(t x)$, is subadditive in $(0, \infty)$, locally bounded above at a point, and there exists an $r_{x} \in(0,1)$ such that $f_{x}\left(r_{x} t\right) \leq r_{x} f_{x}(t)$ for all $t>0$.

For a measure space $(\Omega, \Sigma, \mu)$ denote by $\mathbf{S}=\mathbf{S}(\Omega, \Sigma, \mu)$ the linear space of all the $\mu$-integrable step functions $x: \Omega \rightarrow \mathbb{R}$ and by $\mathbf{S}_{+}$the set of nonnegative $x \in \mathbf{S}$. For a bijection $\varphi: \mathbb{R}_{+} \rightarrow \mathbb{R}_{+}$such that $\varphi(0)=0$ denote by $\mathbb{P}_{\varphi}: \mathbf{S} \rightarrow \mathbb{R}_{+}$ the functional defined by the formula

$$
\mathbb{P}_{\varphi}(x):=\varphi^{-1}\left(\int_{\Omega} \varphi \circ|x| d \mu\right), \quad x \in \mathbf{S} .
$$

In $\S 3$, applying the above result, we give the following characterization of the $\mathbf{L}^{p}$-norm, $p \geq 1$. If $(\Omega, \Sigma, \mu)$ is a measure space with at least two disjoint sets of finite and positive measure and

(1) $\mathbb{P}_{\varphi}(x+y) \leq \mathbb{P}_{\varphi}(x)+\mathbb{P}_{\varphi}(y)$ for all $x, y \in \mathbf{S}_{+} ;$

(2) $\mathbb{P}_{\varphi}(t x) \leq t \mathbb{P}_{\varphi}(x)$, for all $t \in(0,1), x \in \mathbf{S}_{+}$;

then $\varphi(t)=\varphi(1) t^{p}, t \geq 0$, for some $p \geq 1$.

The case when inequalities (1) and (2) are reversed is considered and some complementary results are also presented.

The results of $\S 2$ show that the two basic axioms of seminorms are to some extent dependent. Assuming that a functional $\mathbf{p}$ is subadditive we prove that the second axiom, the homogeneity of $\mathbf{p}$, is then a consequence of a considerably weaker one. In this connection the following natural problem arises. Suppose that $\mathbf{p}$ is (positively) homogeneous. What then can one say about its subadditivity? Answering this question, in $\S 4$, we find a strikingly simple joint generalization of Minkowski's and Hölder's inequalities, which provides us with "one-line"-easy to remember proofs of them.

In the last section we apply our basic result to generalize a Berz theorem on sublinear functions.

\section{SOME PROPERTIES OF SUBADDITIVE AND SUPERADDITIVE FUNCTIONS}

A function $f:(a, \infty) \rightarrow \mathbb{R}, a \geq 0$, is said to be subadditive if

$$
f(s+t) \leq f(s)+f(t), \quad s, t>a .
$$

If the reversed inequality holds then $f$ is said to be superadditive. Since $f$ is superadditive iff $-f$ is subadditive, we deal mainly with subadditive functions.

Lemma 1. Suppose that $f:(a, \infty) \rightarrow \mathbb{R}, a \geq 0$, is subadditive. If $f$ is locally bounded above at a point then there exists $b \geq a$ such that $f$ is locally bounded in $(b, \infty)$.

Proof. Suppose that $f$ is bounded above in a neighbourhood of a point $t_{0}>a$, i.e., that $f(t) \leq M, t \in\left(t_{0}-\varepsilon, t_{0}+\varepsilon\right) \subset(a, \infty)$, for some $M \in \mathbb{R}$ and $\varepsilon>0$. From (1) we have $f(s+t) \leq f(s)+M$ for $t \in\left(s+t_{0}-\varepsilon, s+t_{0}+\varepsilon\right)$, i.e., $f$ is bounded above by $f(s)+M$ in the interval $I_{s}:=\left(s+t_{0}-\varepsilon, s+t_{0}+\varepsilon\right)$. Since the family of intervals $\left(I_{s}\right)_{s>a}$ forms an open covering of the interval $(b, \infty)$ with $b:=a+t_{0}-\varepsilon$, it follows that $f$ is locally bounded above in $(b, \infty)$.

Now let $t_{0}$ denote an arbitrary point of the interval $(b, \infty)$ and let $\varepsilon>0$ be such that $t_{0}-\varepsilon>b$. According to the preceding part of the proof, there is an $M$ such that $f(t) \leq M$ for $t \in\left(t_{0}-\varepsilon, t_{0}+\varepsilon\right)$. Since $t \in\left(t_{0}-\varepsilon, t_{0}+\varepsilon\right)$ if 
and only if $2 t_{0}-t \in\left(t_{0}-\varepsilon, t_{0}+\varepsilon\right)$, we have from (1)

$$
f(t) \geq f\left(2 t_{0}\right)-f\left(2 t_{0}-t\right) \geq f\left(2 t_{0}\right)-M, \quad t \in\left(t_{0}-\varepsilon, t_{0}+\varepsilon\right),
$$

which completes the proof.

Remark 1. It is well known that every subadditive and measurable function $f:(0, \infty) \rightarrow \mathbb{R}$ is locally bounded (cf. [3, Theorem 7.4 .1 , p. $241 ; 11$, Theorem 3.1.1; 4, Theorem 5, p. 406]).

The next lemma is a slight generalization of Theorem 7.6.1 in [3].

Lemma 2. If $f:(a, \infty) \rightarrow \mathbb{R}, a \geq 0$, is subadditive and locally bounded above at a point then

$$
\lim _{t \rightarrow \infty} \frac{f(t)}{t}=\inf _{t>a} \frac{f(t)}{t} .
$$

Proof. By Lemma 1, $f$ is bounded on every compact subset of $(b, \infty)$. Therefore Lemma 2 follows from Theorem 7.6.1 in [3, p. 244] and the remark following its proof.

Now we can prove the main result of this section.

Theorem 1. If $f:(a, \infty) \rightarrow \mathbb{R}, a \geq 0$, is subadditive and locally bounded above at a point, and there exists an $r>1$ such that

$$
f(r t) \geq r f(t), \quad t>a,
$$

then $f(t)=f(1) t$ for all $t>a$.

Proof. Put $\alpha:=\inf _{t>a} f(t) / t$. From (2) by induction we get $f\left(r^{n} t\right) \geq r^{n} f(t)$ for all $n \in \mathbb{N}$ and $t>a$ or, equivalently,

$$
\frac{f(t)}{t} \leq \frac{f\left(r^{n} t\right)}{r^{n} t}, \quad n \in \mathbb{N}, t>a
$$

From Lemma 2, letting $n \rightarrow \infty$, we get $f(t) / t \leq \alpha, t>a$. On the other hand, from the definition of $\alpha$, we have

$$
\frac{f(t)}{t} \geq \alpha, \quad t>a
$$

which completes the proof.

For $a=0$ we obtain

Corollary 1. If $f:(0, \infty) \rightarrow \mathbb{R}$ is subadditive and locally bounded above at a point, and there exists an $r \in(0,1)$ such that $f(r t) \leq r f(t), t>0$, then $f(t)=f(1) t, t>0$.

Remark 2. The assumption of local boundedness above at a point in Lemmas 1 and 2 and Theorem 1 is essential and cannot be replaced even by global boundedness below. To see this take a discontinuous additive function $g: \mathbb{R} \rightarrow$ $\mathbb{R}$ and put $f:=\left.|g|\right|_{(0, \infty)}$. Clearly $f$ is subadditive and bounded below by 0 in $(0, \infty)$. It is well known that $g$ and, consequently, $f$ are not locally bounded at any point. On the other hand one can easily observe that the conclusions of Lemmas 1 and 2 and Theorem 1 for this function $f$ are false.

Remark 3. Replacing $f$ by $-f$ we can reformulate all the above results for superadditive functions. Note that such a procedure is not applicable for a 
function of the type $f:(a, \infty) \rightarrow \mathbb{R}_{+}, a>0$. For example we have the following result (cf. [6]): if $f:(0, \infty) \rightarrow \mathbb{R}_{+}$is superadditive then $f$ is increasing, $\lim _{t \rightarrow 0} f(t)=0$, and

$$
\lim _{t \rightarrow 0} \frac{f(t)}{t}=\inf _{t>0} \frac{f(t)}{t} .
$$

A "dual" counterpart of this result for a subadditive function $f:(0, \infty) \rightarrow \mathbb{R}_{+}$ is obviously false. Let us mention here that Bruckner [2] proved that every superadditive function $f:[0, a] \rightarrow \mathbb{R}$ has the unique minimal superadditive extension $F: \mathbb{R}_{+} \rightarrow \mathbb{R}$ and gave a formula for $F$. In this case a "dual" counterpart holds true.

As an immediate consequence of Theorem 1 we get

Corollary 2. (a) If $f:(a, \infty) \rightarrow \mathbb{R}_{+}, a \geq 0$, is superadditive and there exists an $r>1$ such that $f(r t) \leq r f(t), t>a$, then $f(t)=f(1) t, t>a$.

(b) If $f:(0, \infty) \rightarrow \mathbb{R}_{+}$is superadditive and there exists an $r \in(0,1)$ such that $f(r t) \geq r f(t), t>0$, then $f(t)=f(1) t, t>0$.

Remark 4. If $f:(0, \infty) \rightarrow \mathbb{R}$ satisfies the condition

(i) the function $t \rightarrow f(t) / t$ is nonincreasing in $(0, \infty)$,

then $f$ is subadditive (cf. [3, Theorem 7.2.4, p. 239]). This is a simple criterion of subadditivity. In this connection the following facts may be useful.

(a) If $f:(0, \infty) \rightarrow(0, \infty)$ is continuously differentiable then condition (i) is equivalent to

(ii) $f^{\prime}(t) t \leq f(t), t>0$.

(b) If $f:(0, \infty) \rightarrow(0, \infty)$ is increasing then (i) is equivalent to

(iii) $\log \circ f \circ \exp$ is a nonexpansive mapping of $\mathbb{R}$.

Proof. Take $0<t_{1}<t_{2}$. To prove (a) observe that inequality

$$
\frac{f\left(t_{2}\right)}{t_{2}}<\frac{f\left(t_{1}\right)}{t_{1}}
$$

is equivalent to the inequality

$$
\int_{t_{1}}^{t_{2}} \frac{f^{\prime}(t)}{f(t)} d t \leq \int_{t_{1}}^{t_{2}} \frac{1}{t} d t .
$$

To prove (b) take $u_{1}, u_{2} \in \mathbb{R}$ such that $t_{1}=e^{u_{1}}$ and $t_{2}=e^{u_{2}}$ and note that (3) is equivalent to the inequality

$$
\log f\left(e^{u_{2}}\right)-\log f\left(e^{u_{1}}\right) \leq u_{2}-u_{1} .
$$

Let us finally note the following comparison test of superadditivity. Suppose that $f:(0, \infty) \rightarrow \mathbb{R}_{+}$and $g:(0, \infty) \rightarrow(0, \infty)$. If $g$ is subadditive and $f / g$ is nonincreasing, then $f$ is subadditive.

Proof. For $s, t>0$ we have

$$
\begin{aligned}
f(s+t) & =\frac{f(s+t)}{g(s+t)} g(s+t) \leq \frac{f(s+t)}{g(s+t)}(g(s)+g(t)) \\
& \leq \frac{f(s)}{g(s)} g(s)+\frac{f(t)}{g(t)} g(t)=f(s)+f(t) .
\end{aligned}
$$




\section{AN APPLICATION to a RELAXATION \\ OF THE SEMINORM HOMOGENEITY CONDITION}

Let $(X, \mathbb{R},+, \cdot)$ be a linear space. A set $C \subset X$ is said to be a cone in $X$ if $C+C \subset C$ and $t C \subset C$ for every $t>0$. We have

Proposition 1. Let $(X, \mathbb{R},+, \cdot)$ be a linear space, $C \subset X$ a cone, and $\mathbf{p}: C \rightarrow$ $\mathbb{R}$. Suppose that for every $x \in C$ the function $f_{x}:(0, \infty) \rightarrow \mathbb{R}$, defined by the formula

satisfies the following conditions:

$$
f_{x}(t):=\mathbf{p}(t x), \quad t>0,
$$

(1) $f_{x}$ is subadditive in $(0, \infty)$;

(2) $f_{x}$ is locally bounded above at a point and there exists an $r_{x} \in(0,1)$ such that $f_{x}\left(r_{x} t\right) \leq r_{x} f_{x}(t)$ for every $t>0$.

Then the functional $\mathbf{p}$ is positively homogeneous, i.e., $\mathbf{p}(t x)=t \mathbf{p}(x)$ for every $x \in X$ and $t>0$.

Proof. For every fixed $x \in X$ the function $f:=f_{x}$ satisfies all the conditions of Corollary 1. Consequently we have $f_{x}(t)=f_{x}(1) t, t>0$, i.e., $\mathbf{p}(t x)=t \mathbf{p}(x)$ for all $t>0$.

The next result says in particular that two basic axioms of the seminorm, the triangle inequality and the homogeneity condition, are to some extent dependent. Namely, we have the following

Theorem 2. Let $(X, \mathbb{R},+, \cdot)$ be a linear space and suppose that a functional $\mathbf{p}: X \rightarrow \mathbb{R}$ satisfies the following conditions:

(1) $\mathbf{p}(x+y) \leq \mathbf{p}(x)+\mathbf{p}(y)$ for all $x, y \in X$;

(2) for every $x \in X$ the function $t \rightarrow \mathbf{p}(t x), t>0$, is locally bounded above at a point and there exists an $r=r_{x} \in(0,1)$ such that $\mathbf{p}(r t x) \leq r \mathbf{p}(t x)$ for all $t>0$

(3) $\mathbf{p}(-x)=\mathbf{p}(x)$ for every $x \in X$.

Then $\mathbf{p}$ is a seminorm in $X$.

Proof. Fix an arbitrary $x \in X$ and define $f_{x}:(0, \infty) \rightarrow \mathbb{R}$ by the formula

$$
f_{x}(t):=\mathbf{p}(t x), \quad t>0 \text {. }
$$

From (1) we have

$f_{x}(s+t)=\mathbf{p}((s+t) x)=\mathbf{p}(s x+t x) \leq \mathbf{p}(s x)+\mathbf{p}(t x)=f_{x}(s)+f_{x}(t), \quad s, t>0$,

i.e., $f_{x}$ is subadditive. Since (2) coincides with Proposition 1(2), it follows that

$$
\mathbf{p}(t x)=t \mathbf{p}(x), \quad t>0 .
$$

Hence, making use of (3), we have for $t<0$

$$
\mathbf{p}(t x)=\mathbf{p}((-t)(-x))=-t \mathbf{p}(-x)=|t| \mathbf{p}(x) .
$$

Setting $x=0$ in the inequality of assumption (2), we get $\mathbf{p}(0) \leq r \mathbf{p}(0)$. Since $r<1$, it follows that $\mathbf{p}(0) \leq 0$. On the other hand, from $(1)$ we have $\mathbf{p}(0)=$ $\mathbf{p}(0+0) \leq \mathbf{p}(0)+\mathbf{p}(0)=2 \mathbf{p}(0)$, i.e., $\mathbf{p}(0) \geq 0$. Consequently $\mathbf{p}(0)=0$. Thus we have proved that $\mathbf{p}(t x)=|t| \mathbf{p}(x)$ for all $x \in X$ and $t \in \mathbb{R}$. Now (1), (3), and $\mathbf{p}(0)=0$ imply that $\mathbf{p}(x) \geq 0, x \in X$, which completes the proof.

From Theorem 2 we obtain the following obvious 
Corollary 3. If $(X, \mathbb{R},+, \cdot)$ is a linear space and a functional $p: X \rightarrow \mathbb{R}$ satisfies the following conditions:

(1) $\mathbf{p}(x+y) \leq \mathbf{p}(x)+\mathbf{p}(y)$ for all $x, y \in X$;

(2) $\mathbf{p}(t x) \leq t \mathbf{p}(x)$ for all $t \in(0,1)$ and $x \in X$;

(3) $\mathbf{p}(-x)=\mathbf{p}(x)$ for all $x \in X$,

then $\mathbf{p}$ is a seminorm in $X$.

Remark 5. Both assumptions (2) and (3) of Corollary 3 can be replaced by

$$
\mathbf{p}(t x) \leq|t| \mathbf{p}(x) \text { for all } x \in X \text { and } t \in[-1,1], t \neq 0 \text {. }
$$

In fact, for $t=-1$ we hence get $\mathbf{p}(-x) \leq \mathbf{p}(x)$, which evidently implies that $\mathbf{p}(-x)=\mathbf{p}(x)$.

We generalize this remark as follows.

Theorem 3. Let $(X, \mathbb{R},+, \cdot)$ satisfy the following conditions:

(1) $\mathbf{p}(x+y) \leq \mathbf{p}(x)+\mathbf{p}(y)$ for all $x, y \in X$;

(2) there exists an $r>0$ such that $\mathbf{p}(t x) \leq|t| \mathbf{p}(x)$ for all $x \in X$ and $|t| \leq r, r \neq 0$.

Then $\mathbf{p}$ is a seminorm in $X$.

Proof. It is easily seen that conditions (1) and (2) of Theorem 2 are fulfilled. To prove that condition (3) of Theorem 2 holds true take $n \in \mathbb{N}$ such that $1 / n \leq r$. From our condition (2) we have

$$
\mathbf{p}\left(-\frac{x}{n}\right) \leq \frac{1}{n} \mathbf{p}(x), \quad \mathbf{p}\left(\frac{x}{n}\right) \leq \frac{1}{n} \mathbf{p}(x), \quad x \in X .
$$

Replacing $x$ by $n x$ we get

$$
n \mathbf{p}(-x) \leq \mathbf{p}(n x), \quad n \mathbf{p}(x) \leq \mathbf{p}(n x), \quad x \in X
$$

Hence, making use of (1), we obtain

$$
n(\mathbf{p}(-x)+\mathbf{p}(x)) \leq 2 \mathbf{p}(n x) \leq 2 n \mathbf{p}(x), \quad x \in X,
$$

which implies that $\mathbf{p}(-x) \leq \mathbf{p}(x)$ and, consequently, $\mathbf{p}(-x)=\mathbf{p}(x)$ for all $x \in X$. Thus the theorem follows from Theorem 2 .

In the sequel we consider nonnegative and superadditive functions. Applying Corollary 2(b) we obtain

Proposition 2. Let $(X, \mathbb{R},+, \cdot)$ be a linear space, $C \subset X$ a cone in $X$, and suppose that $\mathbf{p}: C \rightarrow \mathbb{R}_{+}$. If for every $x \in C$ the function $f_{x}:(0, \infty) \rightarrow \mathbb{R}_{+}$, defined by the formula

satisfies the following conditions:

$$
f_{x}(t):=\mathbf{p}(t x), \quad t>0,
$$

(1) $f_{x}$ is superadditive;

(2) there exists an $r=r_{x} \in(0,1)$ such that $f_{x}(r t) \geq r f_{x}(t)$ for all $t>0$, then $\mathbf{p}(t x)=t \mathbf{p}(x)$ for all $x \in C$ and $t>0$.

Since $-\mathbf{p}$ is subadditive and globally bounded above by 0 in $C$, Proposition 2 is also a consequence of Proposition 1.

Remark 6. One can easily check that if $X$ is a real linear space and $\mathbf{p}: X \rightarrow \mathbb{R}$ is superadditive and nonnegative then $\mathbf{p}(x)=0$ for all $x \in X$. To avoid such a triviality we assume in Proposition 2 as well as in the next result that $\mathbf{p}$ is defined in a cone.

As an obvious consequence of Proposition 2 we get 
Theorem 4. Let $(X, \mathbb{R},+, \cdot)$ be a linear space and $C \subset X$ a cone. If $\mathbf{p}: C \rightarrow$ $\mathbb{R}_{+}$satisfies the following conditions:

(1) $\mathbf{p}(x+y) \geq \mathbf{p}(x)+\mathbf{p}(y)$ for all $x, y \in C$;

(2) there exists an $r \in(0,1)$ such that $\mathbf{p}(r x) \geq r \mathbf{p}(x)$ for all $x \in C$,

then $\mathbf{p}(t x)=t \mathbf{p}(x)$ for all $x \in C$ and $t>0$.

Remark 7. Let $X$ be a linear space and $C \subset X$ a cone. Following Rosenbaum [11] we say that a function $\mathbf{p}: C \rightarrow \mathbb{R}$ is quasi-homogeneous if $\mathbf{p}(t x) \leq t \mathbf{p}(x)$ for all $t>1$ and $x \in C$. Theorem 4 implies that every superadditive and quasi-homogeneous function $\mathbf{p}: C \rightarrow \mathbb{R}$ must be positively homogeneous.

\section{AN APPLiCATION TO A CHARACTERIZATION OF THE $\mathbf{L}^{p}$-NORM}

For an arbitrary measure space $(\Omega, \Sigma, \mu)$ denote by $\mathbf{S}=\mathbf{S}(\Omega, \Sigma, \mu)$ the linear space of all the $\mu$-integrable step functions $x: \Omega \rightarrow \mathbb{R}$ and by $\mathbf{S}_{+}=$ $\mathbf{S}_{+}(\Omega, \Sigma, \mu)$ the set of all the nonnegative $x \in \mathbf{S}$. Evidently $\mathbf{S}_{+}$is a cone in S. For a bijection $\varphi: \mathbb{R}_{+} \rightarrow \mathbb{R}_{+}$such that $\varphi(0)=0$ the functional $\mathbb{P}_{\varphi}: \mathbf{S} \rightarrow \mathbb{R}_{+}$, given by the formula

$$
\mathbb{P}_{\varphi}(x):=\varphi^{-1}\left(\int_{\Omega} \varphi \circ|x| d \mu\right), \quad x \in \mathbf{S},
$$

is well defined. Note that if $\varphi$ is of the form $\varphi(t)=c t^{p}, t \geq 0$, for some $c>0$ and $p \geq 1$, then $\mathbb{P}_{\varphi}$ reduces to the $\mathbf{L}^{p}$-norm.

In [5] the present author proved the following

Theorem A. Let $(\Omega, \Sigma, \mu)$ be a measure space with at least two disjoint sets of finite and positive measure, and suppose that $\varphi: \mathbb{R}_{+} \rightarrow \mathbb{R}_{+}$is an arbitrary bijection such that $\varphi(0)=0$. If the functional $\mathbb{P}_{\varphi}$ is a norm in $\mathbf{S}$ then $\varphi(t)=$ $\varphi(1) t^{p}, t \geq 0$, for some $p \geq 1$.

Applying one of the results of the previous section we can prove the following stronger

Theorem 5. Let $(\Omega, \Sigma, \mu)$ be a measure space and $\varphi: \mathbb{R}_{+} \rightarrow \mathbb{R}_{+}$an arbitrary bijection such that $\varphi(0)=0$. If

(1) $\mathbb{P}_{\varphi}(x+y) \leq \mathbb{P}_{\varphi}(x)+\mathbb{P}_{\varphi}(y)$ for all $x, y \in \mathbf{S}_{+}$;

(2) there exists an $r>0$ such that $\mathbb{P}_{\varphi}(t x) \leq t \mathbb{P}_{\varphi}(x)$ for all $x \in \mathbf{S}_{+}, t \in$ $(0, r)$,

then $\mathbb{P}_{\varphi}$ is a norm in $\mathbf{S}$. Moreover, if there are two disjoint sets of finite and positive measure in $\Sigma$ then $\varphi(t)=\varphi(1) t^{p}, t \geq 0$, for some $p \geq 1$.

Proof. Put $X:=\mathbf{S}$ and $\mathbf{p}:=\mathbb{P}_{\varphi}$. Since for every $x \in \mathbf{S}$ we have $|x| \in \mathbf{S}_{+}$ and $\mathbb{P}_{\varphi}(x)=\mathbb{P}_{\varphi}(|x|)$, it follows that the functional $\mathbf{p}=\mathbb{P}_{\varphi}$ satisfies all the assumptions of Theorem 3. Consequently $\mathbb{P}_{\varphi}$ is a norm in $\mathbf{S}$. Now the second statement results from the above quoted Theorem $\mathrm{A}$.

From Theorem 2 we immediately obtain

Theorem 6. Let $(\Omega, \Sigma, \mu)$ be a measure space and $\varphi: \mathbb{R}_{+} \rightarrow \mathbb{R}_{+}$a homeomorphism of $\mathbb{R}_{+}$. If

(1) $\mathbb{P}_{\varphi}(x+y) \leq \mathbb{P}_{\varphi}(x)+\mathbb{P}_{\varphi}(y)$ for all $x, y \in \mathbf{S}_{+}$;

(2) there exists an $r \in(0,1)$ such that $\mathbb{P}_{\varphi}(r x) \leq r \mathbb{P}_{\varphi}(x)$ for all $x \in \mathbf{S}_{+}$, 
then $\mathbb{P}_{\varphi}$ is a norm in $\mathbf{S}$. Moreover, if there are two disjoint sets of finite and positive measure in $\Sigma$ then $\varphi(t)=\varphi(1) t^{p}, t \geq 0$, for some $p \geq 1$.

The second part of this theorem can be generalized as follows.

Theorem 7. Let $(\Omega, \Sigma, \mu)$ be a measure space with two disjoint sets of finite and positive measure and suppose that $\varphi: \mathbb{R}_{+} \rightarrow \mathbb{R}_{+}$is bijective, $\varphi(0)=0$, and $\varphi^{-1}$ is continuous at 0 . If

(1) $\mathbb{P}_{\varphi}(x+y) \leq \mathbb{P}_{\varphi}(x)+\mathbb{P}_{\varphi}(y)$, for all $x, y \in \mathbf{S}_{+}$;

(2) there exists an $r \in(0,1)$ such that $\mathbb{P}_{\varphi}(r x) \leq r \mathbb{P}_{\varphi}(x)$ for all $x \in \mathbf{S}_{+}$, then $\varphi(t)=\varphi(1) t^{p}, t \geq 0$, for some $p \geq 1$.

Proof. According to the assumptions there are $A_{1}, A_{2} \in \Sigma$ such that $A_{1} \cap A_{2}=$ $\varnothing$ and $a_{i}:=\mu\left(A_{i}\right) \in(0, \infty), i=1,2$. Denote by $\chi_{A}$ the characteristic function of a set $A$. Putting in (1) the functions

$$
x:=s \chi_{A_{1}}, \quad y:=t \chi_{A_{2}}, \quad s, t \geq 0,
$$

and making use of the definition of the functional $\mathbb{P}_{\varphi}$, we obtain

$$
\varphi^{-1}\left(a_{1} \varphi(s)+a_{2} \varphi(t)\right) \leq \varphi^{-1}\left(a_{1} \varphi(s)\right)+\varphi^{-1}\left(a_{2} \varphi(t)\right) .
$$

Replacing $s$ by $\varphi^{-1}(s) / a_{1}$ and $t$ by $\varphi^{-1}(t) / a_{2}$, we get

$$
\varphi^{-1}(s+t) \leq \varphi^{-1}(s)+\varphi^{-1}(t), \quad s, t \geq 0 .
$$

Thus $\varphi^{-1}$ is subadditive and, according to the assumptions, bijective and continuous at 0 . In view of the theorem of Swiatkowski and the author [7], $\varphi^{-1}$ is a homeomorphism of $\mathbb{R}_{+}$. Now Theorem 6 concludes the proof.

Applying Theorem 4 and adopting the argument used in the proof of the above quoted Theorem $\mathrm{A}$, we can prove the following

Theorem 8. Let $(\Omega, \Sigma, \mu)$ be a measure space and $\varphi: \mathbb{R}_{+} \rightarrow \mathbb{R}_{+}$an arbitrary bijection such that $\varphi(0)=0$. If

(1) $\mathbb{P}_{\varphi}(x+y) \geq \mathbb{P}_{\varphi}(x)+\mathbb{P}_{\varphi}(y)$, for all $x, y \in \mathbf{S}_{+}$;

(2) there exists an $r>1$ such that $\mathbb{P}_{\varphi}(r x) \leq r \mathbb{P}_{\varphi}(x)$ for all $x \in \mathbf{S}_{+}$,

then $\mathbb{P}_{\varphi}$ is positively homogeneous. Moreover, if there are two disjoint sets of finite positive measure in $\Sigma$ then $\varphi(t)=\varphi(1) t^{p}, t \geq 0$, for some $p \in(0,1]$.

Remark 8. Note that assumption (1) in Theorems 5, 6, and 7 may be replaced by the following considerable weaker one:

$$
\mathbb{P}_{\varphi}(x+y) \leq \mathbb{P}_{\varphi}(x)+\mathbb{P}_{\varphi}(y) \text { for linearly dependent } x, y \in \mathbf{S}_{+} .
$$

The proofs of the so-modified theorems require the use of Proposition 1. Analogously, using Proposition 2, one can strengthen Theorem 8.

Remark 9. In a recent paper [8] the author proved the following converse of Minkowski's inequality. Let $(\Omega, \Sigma, \mu)$ be a measure space with two sets $A, B \in$ $\Sigma$ such that

$$
0<\mu(A)<1<\mu(B)<\infty,
$$

and suppose that $\varphi: \mathbb{R}_{+} \rightarrow \mathbb{R}_{+}$is bijective and $\varphi(0)=0$.

(a) If $\varphi^{-1}$ is continuous at 0 and $\mathbb{P}_{\varphi}(x+y) \leq \mathbb{P}_{\varphi}(x)+\mathbb{P}_{\varphi}(y)$ for all $x, y \in \mathbf{S}_{+}$ then $\varphi(t)=\varphi(1) t^{p}, t \geq 0$, for some $p \geq 1$.

(b) If $\mathbb{P}_{\varphi}(x+y) \geq \mathbb{P}_{\varphi}(x)+\mathbb{P}_{\varphi}(y)$ for all $x, y \in \mathbf{S}_{+}$then $\varphi(t)=\varphi(1) t^{p}, t \geq$ 0 , for some $p \in(0,1]$. 
Thus if condition (4) is satisfied, in most of the results of this section assumption (2) happens to be superfluous.

In this connection there arises a natural problem of the existence of a nonpower bijection $\varphi$ for which the functional $\mathbb{P}_{\varphi}$ is subadditive in $\mathbf{S}_{+}$. Disregarding some trivialities, in view of the converse of Minkowski's inequality, this may happen only if the underlying measure space $(\Omega, \Sigma, \mu)$ satisfies one of the following conditions:

(i) $\mu(A) \leq 1$ or $\mu(A)=\infty$ for every $A \in \Sigma$;

(ii) $\mu(A) \geq 1$ or $\mu(A)=0$ for every $A \in \Sigma$.

In each of these cases one can indicate a broad class of nonpower functions $\varphi$ for which $\mathbb{P}_{\varphi}$ is subadditive or superadditive. We have the following two generalizations of Minkowski's inequality.

Theorem B (First generalized Minkowski's inequality). Suppose that $(\Omega, \Sigma, \mu)$ satisfies condition (i) and $\varphi: \mathbb{R}_{+} \rightarrow \mathbb{R}_{+}$is a homeomorphism of $\mathbb{R}_{+}$two times continuously differentiable.

(a) If $\varphi^{\prime \prime}>0$ in $(0, \infty)$ and $\varphi^{\prime} / \varphi^{\prime \prime}$ is superadditive in $(0, \infty)$, then $\mathbb{P}_{\varphi}(x+y) \leq \mathbb{P}_{\varphi}(x)+\mathbb{P}_{\varphi}(y)$ for all $x, y \in \mathbf{S}$.

(b) If $\varphi^{\prime \prime}<0$ in $(0, \infty)$ and $\varphi^{\prime} / \varphi^{\prime \prime}$ is subadditive in $(0, \infty)$, then $\mathbb{P}_{\varphi}(x+y) \geq \mathbb{P}_{\varphi}(x)+\mathbb{P}_{\varphi}(y)$ for all $x, y \in \mathbf{S}_{+}$.

Example 1. It can easily be verified that $\varphi(t):=t^{2} /(t+1), t \geq 0$, satisfies condition (a) of the above theorem and, clearly, it is not a power function.

Theorem C (Second generalized Minkowski's inequality). Suppose that $(\Omega, \Sigma, \mu)$ satisfies condition (ii) and $\varphi: \mathbb{R}_{+} \rightarrow \mathbb{R}_{+}$is a homeomorphism of $\mathbb{R}_{+}$.

(a) If $\varphi$ and $\log \circ \varphi \circ$ exp are convex, then $\mathbb{P}_{\varphi}$ is subadditive in $\mathbf{S}$.

(b) If $\varphi$ and $\log \circ \varphi \circ \exp$ are concave, then $\mathbb{P}_{\varphi}$ is superadditive in $\mathbf{S}_{+}$.

Example 2. The function $\varphi(t):=e^{t}-1, t \geq 0$, satisfies condition (a) of Theorem $C$. Moreover, taking $(\Omega, \Sigma, \mu)$ such that $\Omega:=\{1,2\}, \Sigma:=2^{\Omega}$, and $\mu(\{1\})=\mu(\{2\}):=1$, we have $x \in \mathbf{S}_{+}$iff $x=x_{1} \chi_{\{1\}}+x_{2} \chi_{\{2\}}, x_{1}, x_{2} \geq 0$, and, by the definition of $\mathbb{P}_{\varphi}$,

$$
\mathbb{P}_{\varphi}(x)=\varphi^{-1}\left(\varphi\left(x_{1}\right)+\varphi\left(x_{2}\right)\right)=\log \left(e^{x_{1}}+e^{x_{2}}-1\right) .
$$

It is easy to verify that we have $\mathbb{P}_{\varphi}(t x) \geq t \mathbb{P}_{\varphi}(x)$ for all $x \in \mathbf{S}_{+}$and $t \in(0,1)$.

Theorem $\mathrm{B}$ has been proved in [8]. Theorem $\mathrm{C}$ is a generalization of Mulholland's inequality (cf. [10], also [3, Theorem 1, p. 201]). Its detailed proof, based on the idea of Mulholland, is given in [9].

Remark 10. Taking above a measure space $(\Omega, \Sigma, \mu)$ with $\Omega:=\{1, \ldots, k\}$, $\Sigma:=2^{\Omega}$, and suitable $a_{i}:=\mu(A i), i=1, \ldots, k$, we obtain the relevant results for the space $\mathbb{R}^{k}$. For instance, from Theorem $\mathrm{C}(\mathrm{a})$ we get: if $\varphi$ is a convex homeomorphism of $\mathbb{R}_{+}$such that $\log \circ \varphi \circ \exp$ is convex then for all $k \in \mathbb{N}$, $a_{i} \geq 1, x_{i} \in \mathbb{R}_{+}, i=1, \ldots, k$, we have

$$
\varphi^{-1}\left(\sum_{i=1}^{k} a_{i} \varphi\left(x_{i}+y_{i}\right)\right) \leq \varphi^{-1}\left(\sum_{i=1}^{k} a_{i} \varphi\left(x_{i}\right)\right)+\varphi^{-1}\left(\sum_{i=1}^{k} a_{i} \varphi\left(y_{i}\right)\right) .
$$




\section{SUBADDITIVITY OF POSITIVELY HOMOGENEOUS FUNCTIONS AND SHORT PROOFS OF MINKOWSKI'S AND HÖLDER'S INEQUALITIES}

We start this section with the following characterization of homogeneous two place functions that are subadditive.

Theorem 9. Suppose that a two place function $\mathbf{p}:(0, \infty)^{2} \rightarrow \mathbb{R}$ is positively homogeneous, i.e., that

$$
\mathbf{p}\left(t x_{1}, t x_{2}\right)=t \mathbf{p}\left(x_{1}, x_{2}\right), \quad t, x_{1}, x_{2}>0,
$$

and let $h:(0, \infty) \rightarrow \mathbb{R}$ be given by $h(t):=p(t, 1)($ or $h(t):=\mathbf{p}(1, t)), t>0$. Then $\mathbf{p}$ is subadditive if and only if $h$ is convex.

Proof. By the homogeneity of $\mathbf{p}$ we have

$$
\mathbf{p}\left(x_{1}, x_{2}\right)=\mathbf{p}\left(\frac{x_{1}}{x_{2}}, 1\right) x_{2}=h\left(\frac{x_{1}}{x_{2}}\right) x_{2}, \quad x_{1}, x_{2}>0 .
$$

Writing the subadditivity of $\mathbf{p}$ we get the inequality

$$
\text { (5) } h\left(\frac{x_{1}+y_{1}}{x_{2}+y_{2}}\right)\left(x_{2}+y_{2}\right) \leq h\left(\frac{x_{1}}{x_{2}}\right) x_{2}+h\left(\frac{y_{1}}{y_{2}}\right) y_{2}, \quad x_{1}, x_{2}, y_{1}, y_{2}>0 \text {, }
$$

which is obviously equivalent to the convexity of $h$. This completes the proof.

As an application to Theorem 9 we obtain

Short proof of Minkowski's inequality. Minkowski's inequality says that the function

$$
\mathbf{p}\left(x_{1}, x_{2}\right):=\left(x_{1}^{p}+x_{2}^{p}\right)^{1 / p}, \quad x_{1}, x_{2}>0,
$$

is subadditive for $p \geq 1$. Thus, in view of Theorem 9, to prove Minkowski's inequality, it is enough to verify that the function

$$
h(t):=\mathbf{p}(t, 1)=\left(t^{p}+1\right)^{1 / p}, \quad t>0,
$$

is convex for $p \geq 1$.

Short proof of Hölder's inequality. Let $p>1$. Hölder's inequality can be obviously written in the form

$$
x_{1}^{1 / p} x_{2}^{1-1 / p}+y_{1}^{1 / p} y_{2}^{1-1 / p} \leq\left(x_{1}+y_{1}\right)^{1 / p}\left(x_{2}+y_{2}\right)^{1-1 / p}, \quad x_{1}, x_{2}, y_{1}, y_{2}>0 \text {, }
$$

which means that the function

$$
\mathbf{p}\left(x_{1}, x_{2}\right):=x_{1}^{1 / p} x_{2}^{1-1 / p}, \quad x_{1}, x_{2}>0,
$$

is superadditive. Thus, according to Theorem 9, to prove Hölder's inequality it is sufficient to observe that the function

$$
h(t):=\mathbf{p}(t, 1)=t^{1 / p}, \quad t>0,
$$

is concave.

Remark 11. The above considerations show that (5) is a joint generalization of Minkowski's and Hölder's inequalities. One can also get $k$-dimensional and an 
integral version of inequality (5). This inequality has been obtained in [6] in a quite different way.

\section{AN APPLICATION TO SUBLINEAR FUNCTIONS}

A function $g: \mathbb{R} \rightarrow \mathbb{R}$ is said to be sublinear if it is subadditive and $g(n t)=$ $n g(t)$ for all $n \in \mathbb{N}$ and $t \in \mathbb{R}$. Berz [1] (cf. also Kuczma [4, p. 415]) proved that every sublinear measurable function $g: \mathbb{R} \rightarrow \mathbb{R}$ has the form

$$
g(t):= \begin{cases}a t, & t \geq 0 \\ b t, & t<0\end{cases}
$$

where $a \geq b$. This result can be generalized as follows.

Theorem 10. If $g: \mathbb{R} \rightarrow \mathbb{R}$ is subadditive, measurable, and there exists an $r>1$ such that $g(r t) \geq r g(t)$ for all $t \in \mathbb{R}$, then $g$ has form (6).

Proof. Since every subadditive and measurable function $g: \mathbb{R} \rightarrow \mathbb{R}$ is locally bounded (cf. Kuczma [4, p. 406]), it follows from Corollary 1 that $f(t)=g(1) t$ for $t \geq 0$ and $g(t)=g(-1) t$ for $t<0$. Setting $a:=g(1), b:=g(-1)$, we get (6).

Remark 12. The above result remains valid on replacing measurability of $g$ by the following condition: $g$ is locally bounded above at a point in $(0, \infty)$ and in $(-\infty, 0)$.

\section{REFERENCES}

1. E. Berz, Sublinear functions on $\mathbb{R}$, Aequationes Math. 12 (1975), 200-206.

2. A. Bruckner, Minimal superadditive extensions of superadditive functions, Pacific J. Math. 10 (1960), 1155-1162.

3. E. Hille and R. S. Phillips, Functional analysis and semigroups, Amer. Math. Soc. Colloq. Publ., vol. 31, Amer. Math. Soc., Providence, RI, 1957.

4. M. Kuczma, An introduction to the theory of functional equations and inequalities, Prace Nauk. Uniw. Śląsk. Katowice, vol. 489, Polish Scientific Publ., Warsaw, Krakow, and Katowice, 1985.

5. J. Matkowski, On a characterization of $L^{p}$-norm, Ann. Polon. Math. 50 (1989), 137-144.

6. __ Functional inequality characterizing convex functions, conjugacy and a generalization of Hölder's and Minkowski's inequalities, Aequationes Math. 40 (1990), 168-180.

7. J. Matkowski and T. Swiątkowski, Quasi-monotonicity, subadditive bijections of $\mathbb{R}_{+}$and characterization of $L^{p}$-norm, J. Math. Anal. Appl. 154 (1991), 493-506.

8. J. Matkowski, The converse of the Minkowski's inequality theorem and its generalization, Proc. Amer. Math. Soc. 109 (1990), 663-675,

9. __ A generalization of Mulholland inequality (submitted).

10. H. P. Mulholland, On generalizations of Minkowski's inequality in the form of a triangle inequality, Proc. London Math. Soc. (2) 51 (1950), 294-307.

11. R. A. Rosenbaum, Sub-additive functions, Duke Math. J. 17 (1950), 227-242.

Department of Mathematics, Technical University, 43-309 Bielsko-Biala, Poland 